\title{
PMAC: Probabilistic Multimodality Adaptive Control
}

\author{
R. Herzallah and D. Lowe \\ Mathematics Group, Aston University, Aston Triangle, Birmingham B4 7ET. UK.
}

\begin{abstract}
This paper develops a probabilistic multimodal adaptive control approach for systems that are characterised by temporal multimodality where the system dynamics are subject to abrupt mode switching at arbitrary times. In this framework the control objective is redefined such that it utilises the complete probability distribution of the system dynamics. The derived probabilistic control law is thus of a dual type that incorporates the functional uncertainty of the controlled system. A multi-modal density model with prediction errordependent mixing coefficients is introduced to effect the mode switching. This approach can deal with arbitrary noise distributions, nonlinear plant dynamics and arbitrary mode switching. For the affine systems focussed upon for illustration in this paper the approach has global stability. The theoretical architecture constructs are verified by validation on a simulation example.
\end{abstract}

\section{Introduction}

In this article, we propose a new adaptive probabilistic control framework for analysing and controlling complex dynamical systems capable of switching randomly between regimes. The aim of the probabilistic adaptive controller is to estimate the control signals to define a regime of operation of the dynamical system and force its dynamics to converge to a predefined desired behaviour specified by multiple performance criteria. We devise a generalisation of the Mixture Density Network approach, modified to be a Multi-Modal Density Model (MMDM) with prediction error-dependent mixing coefficients. The mixing coefficients are used to select the most probable mixture mode and produces globally stable solutions in the affine model case considered in this

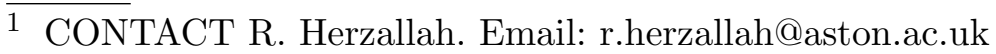


article. The MMDM estimates the arbitrary conditional distributions of the systems' dynamics that are of interest to the control problem and can switch immediately and effectively from one mode of operation to another. The fully probabilistic approach leads us to redefine the control objective to incorporate the probability distribution of the system dynamics and so is of the dual type.

The architecture proposed in this paper circumvents several issues of previous multi-objective control approaches which are often constrained to be linear, deterministic and unable to exploit system uncertainties. The set of new features offered as part of this architecture include:

- a redefined control objective such that the optimal controller should make the pdf of the tracking error as narrow as possible. In addition, it should minimise the mean value of the tracking error and control energy.

- a multimodal density function (MMDN) which is used to estimate the arbitrary distribution of the dynamic generator of the data such that it accommodates the sudden environmental changes and switches.

- the mixing coefficients of this MMDN depend on the prediction error, where we use a neural network to estimate the mixing coefficients from the prediction error and another neural network to predict the means and variances of the MMDN Gaussian kernel functions given the input state vector.

- a learning algorithm in which a softmax normalisation of the mixing coefficients is used to competitively divide up the module learning and selection problem.

- the use of the most probable component at an instant derives an explicit closed form solution for the optimal control demonstrated in the case of nonlinear affine systems.

This combination of features allows the proposed architecture of the MMDN to learn multiple modes of operation and switch between them based on prediction errors between estimated and actual modes of operation, and thus can estimate an arbitrary distribution of random signals.

Although several approaches to multi-objective control have been developed only a few of them have considered design methods for the controller parameters. In addition, most follow deterministic design approaches which are unable to deal with the general control problem where high dimensionality, multi-modality and uncertainty are involved.

The adaptive probabilistic multi-objective framework proposed here belongs to the multi-model framework [Fabri and Kadirkamanathan, 2001, Herzallah, 2012] where the control system is described by dynamic probabilistic mixture models and neural networks. Historical process data that describes the dynamics of the system to be controlled are analysed by a Bayesian approach to build the mixture model in terms of a linear combination of adaptive dynam- 
ical kernel functions. The estimation of the proposed probabilistic adaptive control framework is based on the use of mixture density network [Bishop, 1995, Herzallah and Lowe, 2003], that is extended and developed in this work to handle uncertain jump systems. This method of estimation is referred to as the multiple mode density network. The proposed mixture model provides a general framework for modelling the conditional distribution of the system's dynamics that accommodates non-Gaussian noise and multi-modality in the system dynamics. Within this setting each modality is associated with one mixture component. The weight of the mixture components on the other hand represents the probability of occurrence of a particular modality of operation. Moreover, the means, variances and priors of the mixture components are non-linear functions of the system's input and estimation error. This description of mixtures of a system is especially useful for complex large scale control systems when the system behaviour exhibits several different modalities of operation. Examples of such systems include a multi-modal distribution in a quality characteristics or the sporadic shift of the process average due to sporadic adjustment of a machine parameters, the transition from free flowing traffic to grid-lock in traffic networks, and the inverse kinematics solution of robotics systems. Although we propose the use of neural networks for the estimation of the mixture model kernel function parameters in the multiple model density network, the use of neural networks is not mandatory and any other nonlinear universal function approximator can be used instead.

To design a control strategy, the probabilistic indirect adaptive control methodology [Duarte, 1996, Kersting, 2017] is adopted. Indirect adaptive control is concerned with the minimisation problem of the tracking error as measured between the desired and actual output of the system. However, in their conventional form these methods aim at the minimisation of the mean of the tracking error, thus they ignore knowledge of uncertainty and only derive heuristic certainty equivalence controllers. In contrast to these methods the probabilistic description of a mixture of Gaussians of the controlled system is used in this work to derive the probability distribution of the tracking error and derive control laws that aim at minimising the width and mean of the tracking error in addition to the minimisation of the control energy, thus yielding a novel dual probabilistic adaptive controller. This aim of simultaneous minimisation of the mean and variance of the tracking error and control energy provides a multiperformance criteria and defines the multiobjective problem considered in this work. This natural definition of the multiperformance index, makes the proposed framework distinct to other multiobjective [Peitz and Dellnitz, 2018] as well as multiple model [Apkarian et al. 2014, Piguet et al., 1997] approaches that are still suffering from the lack of a systematic methodology that guarantees a satisfactory solution to the stochastic nonlinear and uncertain multimodal problem.

To reemphasise, the reformulated aim of the control objective as defined in 
this work accompanied with the multiple model density network provides a novel and distinct context to handle the multiobjective multiple modes control problem and allows for the derivation of a novel dual controller that takes a model's functional uncertainty into consideration.

The organisation of the paper is as follows. The objective functional of probabilistic multi-modal control along with its motivation are given in Section 2 . Section 3 introduces the type of system model, discusses the multiple mode density network, and defines the control objective. The main results including the derivation of both of the conditional density function based and most probable component based probabilistic model adaptive controllers are give in Section 4. A simulation study is presented in Section 5. Section 6 gives concluding remarks.

\section{Objective Functional of Probabilistic Multi-modal Control}

\subsection{Motivation of Probabilistic Multi-modal Adaptive Control}

Probabilistic Adaptive Control is an active field in the design of control systems to deal with uncertainties. Traditionally this method has been based on single fixed or slowly adapting models of the system. While the single model adaptive control method has proven to be efficient for dealing with low levels of systems' uncertainties, it performs poorly in more complex systems that are characterised by high levels of uncertainty such as multimodality, hysteresis, and that operate in multiple environments. To cope with such situations, the multiple model adaptive control method was introduced in the early 1990s [Fekri et al., 2006, Leith et al., 2003, Narendra and Chen, 2000]. In the context of multiple model adaptive control, several switching mechanisms have been discussed. Examples include direct switching [Fu and Barmish, 1986, Zhivoglyadov et al., 2000] where the choice of the switching was taken to be dependent upon the output of the plant. Indirect switching where multiple models were used to determine when and to which controller to switch to was then proposed in [Middleton et al., 1988, Narendra et al., 1995, Narendra and Chen, 2000] to overcome the impracticality of the direct switching methods. Data driven unfalsified adaptive control methods which seek evidence in the system output data to switch a controller out of the loop when the controller fails to achieve the objective of the control system was proposed in [Safonov and Tsao, 1997]. Dwell time switching logic [Morse, 1996, Morse, 1997] and Hysteresis based switching logic [Hespanha et al., 2003] were also proposed to address the problem of how to coordinate the switching. Many of these methods have been developed in an attempt to define minimal prior information to achieve good and robust performance of the controlled 
systems. In a pursuit to try and minimise the number of required models, new concepts in adaptive multiple control that are based on the estimation of multiple models that cooperate together were proposed in [Han and Narendra, 2012]. In many of the aformentioned works, only the deterministic description of the controlled systems dynamics were considered. The design of multiple model adaptive control laws for stochastic dynamical systems has received little attention and was mostly limited to the minimisation of the mean value of the tracking error between the actual output of the system and a predefined desired output, hence they fail to account for parameter and functional uncertainties of the controlled system and can only derive heuristic certainty equivalence control laws [Narendra and Chen, 2000, Watanabe and Tzafestas, 1989, Zhang et al., 2012]. In addition, multiple adaptive control methods are commonly restricted to control linear state space switching systems [Fiacchini et al., 2016, Fiacchini and Tarbouriech, 2017, Zhang, 2012] rather than considering the general realistic problem of non-linear control.

This lack of a general framework that guarantees the derivation of optimal controllers which consider the inherent nonlinearity and uncertainty of controlled systems drives the work in the current paper. In particular, we develop a general framework for minimising the uncertainty of the conditional probability distribution of the tracking error, which also accounts for systems' uncertainty and multimodality in the control system design. Mixture density networks (MDNs) were proposed in the literature to approximate the arbitrary distribution of the generator of the systems' data, and was extended to deal with dynamical control systems for the first time in [Herzallah and Lowe, 2003]. However, the extended MDN as proposed in [Herzallah and Lowe, 2003] can only handle dynamical systems that are characterised by spatial multimodality where the system dynamics are highly complex and operate in different operating space. For the dynamical systems considered in this paper where the system dynamics are characterised by temporal multimodality and where the dynamics change abruptly and arbitrarily in time the extended MDN as proposed in [Herzallah and Lowe, 2003] will not yield good performance. Consequently, this paper introduces a novel Multiple Mode Density network (MMDN) that can estimate the arbitrary conditional distributions of the systems' dynamics that are of interest to the control problem and can switch immediately and effectively from one mode of operation to another. Hence, the proposed research proposes a novel and efficient methodology where the multimodal control law is obtained from the multiple components of the forward dynamical model and designed to deal with the uncertainty, complexity and multimodality of the system operation. In the proposed framework, the switching between different modes of operation is based on indirect methods where the switching is achieved through mixing coefficients that are calculated based on the error between the actual and estimated outputs of the system. 


\subsection{Objective Functional}

In this section we will formulate the objective functional of the probabilistic multimodal switching control problem. The formulation will be developed for general stochastic control problems where due to the effect of random forces on the system output, $\mathrm{y}_{t+d}$ the behavioral dynamics of the system can only be specified by the probability distribution of its output conditioned on its input $u_{t}$, and its measurable state vector $x_{t}=\left[\mathrm{y}_{t-1}, \mathrm{y}_{t-2}, \ldots, \mathrm{y}_{t-n}, u_{t-1}, \ldots, u_{t-m}\right]$. This conditional probability distribution of the system output can be described in the following general form,

$$
P_{\mathrm{y}}\left(\mathrm{y}_{t+d} \mid u_{t}, x_{t}\right)
$$

where $d$ is the relative degree of the system, $n$ is the maximum delay in the output, and $m$ is the maximum delay in the input. In general $P_{\mathrm{y}}$ is an arbitrary conditional probability density function of the system dynamics which can have different numbers of multimodalities depending on the operating region of the system.

To facilitate the development of the general methodology of minimising the uncertainty of the conditional probability distribution of the tracking error, the density of the tracking error needs to be acquired. This can be obtained from the density of the system output, $\mathrm{y}_{t+d}$ as follows,

$$
P_{e}\left(\mathrm{y}_{t+d}, \mathrm{y}_{t+d}^{r}\right)=P_{\mathrm{y}}\left(e_{k+d}, \mathrm{y}_{t+d}^{r}\right)
$$

where $e_{k+d}=\mathrm{y}_{t+d}-\mathrm{y}_{t+d}^{r}$ is the tracking error, and $\mathrm{y}_{t+d}^{r}$ is the desired output of the system. Hence, $P_{e}$ is also an arbitrary conditional probability density function which can have different numbers of multimodalities depending on the complexity of the error surface.

Note that in this formulation, the value of the control input $u_{t}$ indirectly parameterises and affects the distribution of the tracking error through its effect on the distribution of the system output, $P_{\mathrm{y}}$. To best accommodate the complexity and stochastic nature of the control system, we aim in this paper to design randomised controllers, that are naturally explorative and that can handle high level of uncertainty and multimodality in the system dynamics. The details of the design method of the randomised controller will be discussed later in the paper. However, this will essentially follow our principle for the design problem, which can be stated as follows:

A controller of a stochastic system shapes the probability density function of its tracking error. The control aim is that the optimal controller should make the pdf of the tracking error as narrow as possible. In addition, it should minimise the mean value of the tracking error and control energy. 
Remark. A narrow distribution indicates that the uncertainty of the tracking error is small which also corresponds to a small variance for the model in this paper.

Having accepted this rephrased control aim, it is reasonable to formulate the following objective functional for the purpose of the controller design,

$$
\begin{aligned}
J\left(u_{t}\right) & =R \int\left\|e_{t+d}-<e_{t+d} \mid, \mathrm{y}_{t+d}, \mathrm{y}_{t+d}^{r}>\right\|^{2} P_{e}\left(\mathrm{y}_{t+d}, \mathrm{y}_{t+d}^{r}\right) d e_{t+d} \\
& +M\left(\int e_{t+d} P_{e}\left(\mathrm{y}_{t+d}, \mathrm{y}_{t+d}^{r}\right) d e_{t+d}\right)^{2}+Q u_{t}^{2}
\end{aligned}
$$

where $R, M, Q$ are constants reflecting the relative importance of the various terms. To summarize, a novel MMDN will be developed to estimate the general distribution that captures the complex and arbitrary structure of the tracking error and that can switch mode abruptly and arbitrarily in time. Similar to the MDN, the MMDN estimates the parameters of the mixture model as continuous single-valued functions of the input variables using a standard feed-forward neural network. On the other hand, in contrast to other standard multiple model approaches, the MMDN minimises an error function that is constructed from the likelihood of the linear combination of mixture models. This new architecture which we refer to as the MMDN will be discussed in detail in the next section. An important characteristic of the MMDN is that it is able to produce a conditional density where the number of multimodalities in this density function depends on the surface of the tracking error at the control instant $t$. The randomised controllers are then optimised such that the uncertainty of the conditional distribution of the tracking error is minimised and the derived control law is of general dual type as opposed to the heuristic certainty equivalence type. The current paper proposes a general framework for solving this optimisation problem in a computationally coherent manner. The basic idea of the proposed framework is that a control law exists to control the multimodal system where the inverse controller is derived such as to minimise the mean value of the tracking error and at the same time make its probability density function as narrow as possible. The combination of the inverse controller and forward model of the system dynamics constitutes the closed loop behavior of the controlled dynamical system. It is described in terms of the general probability density function of the system forward dynamics and tracking error represented by a mixture of Gaussian models. Each Gaussian component of the tracking error density function evaluates a number of probable control signals, and the component generating the minimum width and mean of the tracking error is used to represent the current operating mode of the system and derive the most suitable control signal to control the system. This overall framework is referred to as Probabilistic Multimodal Adaptive Control (PMAC). This is especially useful for controlling systems that are characterised by high levels of uncertainty, multimodality, 
and hysteresis.

\section{System Model and Control Design}

The objective functional of the PMAC developed in the previous section will be implemented here to demonstrate the design of a randomised controller to control an affine class of jump nonlinear stochastic single input single output discrete time systems described by the following general form,

$$
\begin{aligned}
\mathrm{y}_{t+d} & =f_{t+d}+g_{t+d} u_{t}, \\
f_{t+d} & =f_{m(t+d)}\left[x_{t}\right]+\eta_{t+d}, \\
g_{t+d} & =g_{m(t+d)}\left[x_{t}\right]+\eta_{t+d} .
\end{aligned}
$$

where $\mathrm{y}_{t+d}, u_{t}$, and $x_{t}$ are the system output, control input vector, and input state vector respectively as defined earlier, $f_{m(t+d)}\left[x_{t}\right]: \mathbb{R}^{n+m} \longmapsto \mathbb{R}$ and $g_{m(t+d)}\left[x_{t}\right]: \mathbb{R}^{n+m} \longmapsto \mathbb{R}$ are smooth unknown non-linear functions of the state, and $\eta_{t+d}$ is an additive Gaussian noise signal. The smooth non-linear functions $f_{t+d}$ and $g_{t+d}$ could switch form at an arbitrary instant in time according to the switching index $m(t+d) \in\{1,2, \ldots, H\}$. Here $H$ represents the number of modes of operation that the system can assume during the course of time. In addition, the probability density function of the system output $\mathrm{y}_{t+d}$ are assumed to be unknown and need to be estimated.

The task of controlling such system is challenging, due to the presence of both mode jumps, which is a form of temporal multimodality, in addition to the inherent uncertainty of the dynamics of the modes. The latter refers to the functional uncertainty in the system model and the inaccuracy in identifying prior accurate models for all modes. As stated by the objective functional defined in section 2.2, the objective of the control design is to make the probability density function of the tracking error between the system and desired outputs as narrow as possible. This design objective will be achieved under the following assumptions:

Assumption 1. The dynamics of every mode are minimum phase and the functions $g_{m(t+d)}$ are bounded away from zero.

Assumption 2. The functions $f_{m(t+d)}\left[x_{t}\right]$ and $g_{m(t+d)}\left[x_{t}\right]$ are smooth nonlinear functions.

Assumption 3. The random signals $f_{t+d}$ and $g_{t+d}$ are stochastically independent.

Assumption 4. The noise $\eta_{t+d}$ is an independent and identically distributed Gaussian noise signal that has unknown probability density function.

As will be seen later, the proposed PMAC based on MMDN in this paper 
provides an efficient framework for addressing both of the aforementioned challenges. Within this framework $H$ Kernel functions in the MMDN, one per mode, are used to identify the nonlinear mode dynamics of the jump system which are then used to derive optimal control laws via the indirect adaptive control method. As will be discussed in Section 3.1, we develop an automatic mode detection approach based on the identification error between the active mode output and the output of the Kernel functions to switch between the $H$ Kernel functions in the MMDN and force a particular Kernel function to learn, by online adjustment of its parameters, the dynamics of that mode. More significantly, the estimated Kernel functions of the MMDN are used to derive original multiple dual adaptive control laws that guarantee robust control results for the uncertain temporal multimodal systems considered in this paper. The proposed dual adaptive control scheme is facilitated by our definition of the objective functional (3) that contains terms related to the estimated variance of the tracking error and to penalise the control input, thus yielding a control law that is not of the heuristic certainty equivalence type. To re-emphasise, this general multiple model framework is capable of handling dynamic uncertainty and multimodality of the control system. The problem of controlling temporal multimodal nonlinear systems has been considered in the literature, but has only covered deterministic or linear systems. The proposed framework on the other hand utilises the probabilistic information from a set of kernel functions estimated by the MMDN and the automatic mode detection to derive an optimal control law that can switch between active modes and at the same time takes knowledge of uncertainty into consideration for more robust control results.

\subsection{Multiple Mode Density Network, MMDN}

The class of dynamical systems defined in Equation (4) is characterised by temporal multimodality. For these systems, the new system parameters must be tracked fast enough to guarantee small transient degradation in control performance. Multiple models were proposed in the literature to provide a solution for this problem. The problem is also related to fault detection and control reconfiguration [Boskovic and Mehra, 2018, Liu et al., 2018]. However, these methods are based on the certainty equivalence assumption, thus do not provide the optimal solutions for stochastic systems. Utilising the benefits of a modular approach, we propose a new architecture of a mixture of Gaussian densities network where the mixing priors are functions of the predicted error thus emphasising individual modes, which we refer to as an MMDN. The main objective of the MMDN network is to estimate the arbitrary distribution of the dynamic generator of the data such that it accommodates the sudden environmental changes and switches. To explain the general architecture and operation concept of the MMDN, let $h_{t+d}$ represents either $f_{t+d}$ or $g_{t+d}$ of 
Equation (4), then the general distribution of $h_{t+d}$ can be estimated as follows,

$$
P\left(h_{t+d} \mid x_{t}, e_{h_{t}}\right)=\sum_{i=1}^{K} \varrho_{i}\left(e_{h_{t}}\right) \varphi_{i}\left(h_{t+d} \mid x_{t}\right)
$$

where $\varrho_{i}\left(e_{h_{t}}\right)$ represent the mixing coefficients of $h_{t+d}$, and can be regarded as prior probabilities (which depend on the error vector between the actual, $h_{t}$ and estimated, $\bar{h}_{t ; i}$ outputs at time $\left.t, e_{h_{t}}^{i}=h_{t}-\bar{h}_{t ; i}\right) ; \varphi_{i}\left(h_{t+d} \mid x_{t}\right)$ are the kernel distributions of the mixture model of $h_{t+d}$ (whose parameters are conditioned on the input state vector, $x_{t}$ ), and $K$ is the number of kernels in the mixture models, which is also the number of modes $H$ assumed in the dynamics. Various choices are available for the kernel functions, but this work uses spherical Gaussians of the form,

$$
\varphi_{i}\left(h_{t+d} \mid x_{t}\right)=\frac{1}{(2 \pi)^{c / 2} \kappa_{i}^{c}\left(x_{t}\right)} \exp \left(-\frac{\left\|h_{t+d}-\bar{h}_{i}\left(x_{t}\right)\right\|^{2}}{2 \kappa_{i}^{2}\left(x_{t}\right)}\right)
$$

where $c$ is the dimensionality of the random signal $h_{t+d}, \bar{h}_{i}\left(x_{t}\right)$ represents the centre of the $i t h$ kernel, with components $\bar{h}_{i j}$. In Equation (6), the components of the output variable are assumed to be statistically independent within each of the kernel functions, and can be described by a variance $\kappa_{i}^{2}\left(x_{t}\right)$.

The mixture model defined in Equation (5) provides a general formalism for estimating an arbitrary conditional density function $P\left(h_{t+d} \mid x_{t}, e_{h_{t}}\right)$ of the random signal $h_{t+d}$. The dependency of the mixing coefficients on the error signal between actual and estimated outputs as proposed in the MMDN allows the switching between different modalities where the onset of the mode switch and the currently active mode do not depend on any of the measurable state variables. This dependency of the mixing coefficient on the error signal, rather than the state vector as in the enhanced version of the MDN, also means that two neural networks are required to estimate the various parameters of the mixture model. The first neural network, which we refer to as the kernel functions network, takes the means $\bar{h}_{i}\left(x_{t}\right)$ and the variances $\kappa_{i}^{2}\left(x_{t}\right)$ as its outputs and the state vector $x_{t}$ as its input. The second neural network, which we refer to as the mixing coefficients network, takes the mixing coefficients $\varrho\left(e_{h_{t}}\right)$ as its outputs and the error vector $e_{h_{t}}^{i}$ as its input. The architecture of the MMDN is shown in Figure 1.

To ensure that the two neural networks of the MMDN learn to competitively divide up experience and solve the module learning and selection problems, the ith mixing coefficient of the $i t h$ kernel component is calculated by transforming the predicted error value from that component by the softmax function, 


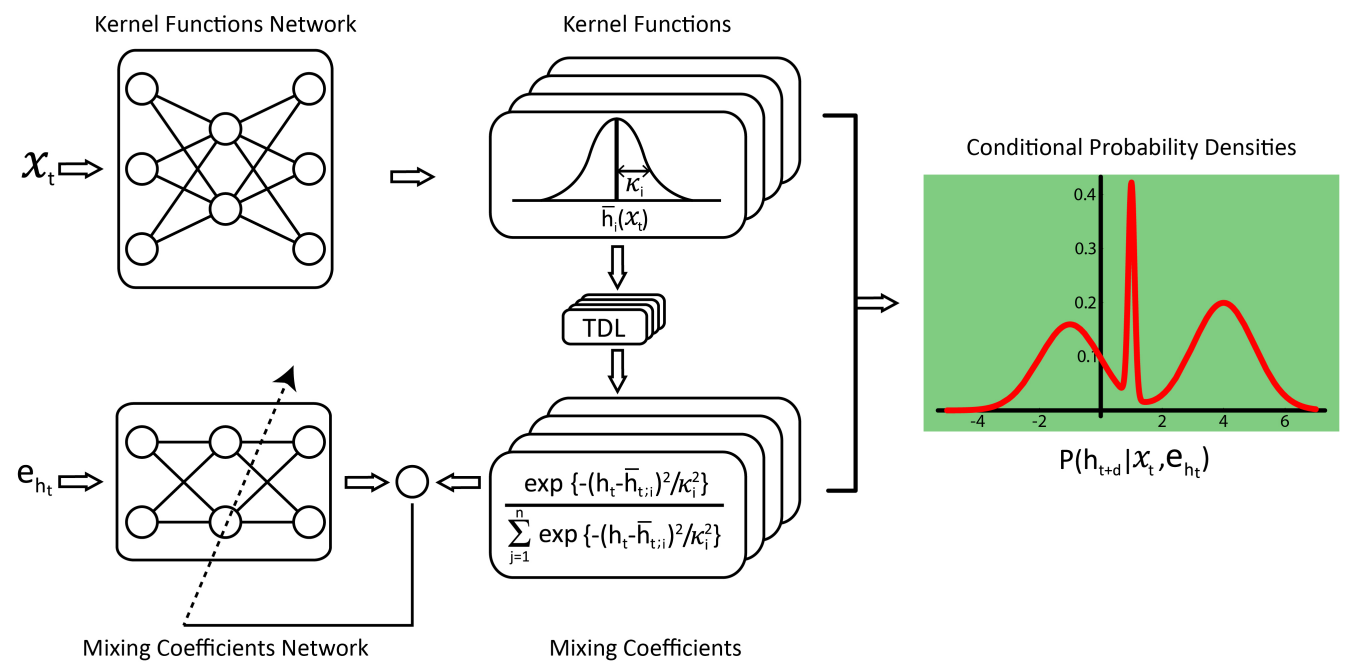

Figure 1. The architecture of the proposed multiple model density network, MMDN. The general conditional probability densities $P\left(h_{t+d} \mid x_{t}, e_{h_{t}}\right)$ are represented as a parametric model of kernel functions and mixing coefficients. The parameters of the kernel functions are determined by the outputs of the Kernel Functions network that takes $x_{t}$ as its input vector. A second standard neural network, mixing coefficient networks, that takes the error vector between the estimated and actual mixing coefficients $e_{h_{t}}$ is also implemented to provide a prediction for the mixing coefficient and switch between various kernel components based on the current active mode of the dynamical system. TDL is time delay lines.

$$
\tilde{\varrho}_{i}\left(e_{h_{t}}\right)=\frac{\exp \left\{-\left(h_{t}-\bar{h}_{t ; i}\right)^{2} / \kappa_{i}^{2}\right\}}{\sum_{j}^{M} \exp \left\{-\left(h_{t}-\bar{h}_{t ; j}\right)^{2} / \kappa_{j}^{2}\right\}},
$$

which gives higher mixing coefficients for those components which capture the current behavior of the dynamical system. These mixing coefficients are then compared to the estimated coefficients generated from the transformed outputs of the mixing coefficients neural network by the softmax function. These error signals between the estimated and actual mixing coefficients can hence be used to update the weights of the mixing coefficients network. The predicted mixing coefficients from the mixing coefficients network are then used to control the learning of the kernel functions network using an error function from the likelihood as follows,

$$
E=-\sum_{i=1}^{M} \varrho_{i}\left(e_{h_{t}}\right) \varphi_{i}\left(h_{t+d} \mid x_{t}\right)
$$

This definition of the error function of the kernel functions network as the likelihood function implies that the kernel components with high mixing coefficients receive more contribution than components with low mixing coefficients which in turn allows the efficient division of the learning experience 
among the kernel components. In addition, the use of the predicted mixing coefficients rather than the actual coefficients allows the components to switch between them before the generation of the control action and the evaluation of its consequences.

To summarise, the proposed architecture of the MMDN can learn multiple modes of operation and switch between them based on prediction errors between estimated and actual mode of operation, thus can estimate an arbitrary distribution of random signals.

\subsection{Control Objective}

The control objective as stated in Section 2 is to design a randomised controller that minimises the uncertainty of the conditional distribution of the tracking error function. As discussed earlier, the tracking error distribution can be obtained from the distribution of the forward dynamics of the plant. The developed architecture of the MMDN in Section 3.1 will be used here to provide an estimate for the output pdf of the system defined in (4). To do this we note that the output of the system of (4) is the addition of a multimodal non-linear function $f_{t+d}$ to the product of another multimodal non-linear function $g_{t+d}$ with the control input $u_{t}$. Thus, two MMDNs are required to estimate the pdfs of the two non-linear multimodal functions $f_{t+d}$ and $g_{t+d}$. As discussed in Section 3.1, the outputs of the two MMDNs approximating the conditional distributions of the non-linear functions $f_{t+d}$ and $g_{t+d}$ are respectively given by,

$$
\begin{aligned}
& P\left(f_{t+d} \mid x_{t}, e_{f_{t}}\right)=\sum_{j=1}^{M_{1}} \alpha_{j}\left(e_{f_{t}}\right) \phi_{j}\left(f_{t+d} \mid x_{t}\right), \\
& P\left(g_{t+d} \mid x_{t}, e_{g_{t}}\right)=\sum_{i=1}^{M_{2}} \beta_{i}\left(e_{g_{t}}\right) \theta_{i}\left(g_{t+d} \mid x_{t}\right),
\end{aligned}
$$

where $\alpha_{j}\left(e_{f_{t}}\right)$ and $\beta_{i}\left(e_{g_{t}}\right)$ represent the mixing coefficients of $f_{t+d}$ and $g_{t+d}$ respectively, $\phi_{j}\left(f_{t+d} \mid x_{t}\right)$ and $\theta_{i}\left(g_{t+d} \mid x_{t}\right)$ are the kernel distributions of the mixture models of $f_{t+d}$ and $g_{t+d}$ respectively, and $M_{1}$ and $M_{2}$ are the number of kernels in the mixture models. The kernel functions of both MMDNs are given by spherical Gaussian functions as follows,

$$
\begin{aligned}
& \phi_{j}\left(f_{t+d} \mid x_{t}\right)=\frac{1}{(2 \pi)^{c / 2} \sigma_{j}^{c}\left(x_{t}\right)} \exp \left(-\frac{\left\|f_{t+d}-\mu_{j}\left(x_{t}\right)\right\|^{2}}{2 \sigma_{j}^{2}\left(x_{t}\right)}\right), \\
& \theta_{i}\left(g_{t+d} \mid x_{t}\right)=\frac{1}{(2 \pi)^{c / 2} \rho_{i}^{c}\left(x_{t}\right)} \exp \left(-\frac{\left\|g_{t+d}-\nu_{i}\left(x_{t}\right)\right\|^{2}}{2 \rho_{i}^{2}\left(x_{t}\right)}\right)
\end{aligned}
$$


where $c$ is the dimensionality of the random signals $f_{t+d}$ and $g_{t+d}, \mu_{j}\left(x_{t}\right)$ and $\nu_{i}\left(x_{t}\right)$ represent the centres of the $j t h$ and $i t h$ kernel, with components $\mu_{j k}$ and $\nu_{i k}$. The following conditions, related to the MMDNs, are assumed to hold:

Assumption 5. The state $x_{t}$ is always confined within a bounded region of state space $x_{t} \in X$.

Assumption 6. For every local mode of operation $m(t+d)$, there exist some optimal Kernel centres, and variances as well as mixing coefficients that are capable of approximating the probability density functions of the system mode dynamics $\left(f_{m}(t+d), g_{m}(t+d)\right)$ within the region $X$.

Assumption 6 follows from the universal approximation property of the MDN. Both of the assumptions 5 and 6 ensure that the system mode activity can be captured by one of the Kernel functions of the estimated pdf of the system output.

By assumption 3 it follows that the conditional probability density function of the system output defined in Equation (4) can be obtained as follows

$$
\begin{aligned}
P_{\mathrm{y}}\left(\mathrm{y}_{t+d} \mid x_{t}, e_{f_{t}}, e_{g_{t}}\right) & =P_{f} * P_{u g} \\
& =P\left(f_{t+d} \mid x_{t}, e_{f_{t}}\right) * P\left(u_{t} g_{t+d} \mid x_{t}, e_{g_{t}}\right),
\end{aligned}
$$

where $P_{f} * P_{u g}$ denotes the convolution of $P_{f}$ and $P_{u g}$. Given the forms of the probability density functions of the random signals $f_{t+d}$ and $g_{t+d}$ defined in Equations (9) and (10) respectively, the above convolution of the two pdfs $P_{f}$ and $P_{u g}$ can be shown to be given by the form stated in the following lemma. Lemma 3.1. Subject to assumption 3, the forms of pdfs of the random signals $f_{t+d}$ and $g_{t+d}$ defined in Equations (9) and (10) respectively, and the forms of Gaussian kernels defined in Equations (11) and (12), the pdf of the system output defined in Equation (13) is given by

$$
\begin{aligned}
P_{\mathrm{y}}\left(\mathrm{y}_{t+d} \mid x_{t}, e_{f_{t}}, e_{g_{t}}\right) & =\sum_{j=1}^{M 1} \sum_{i=1}^{M_{2}} \alpha_{j}\left(e_{f_{t}}\right) \phi_{j}\left(f_{t+d} \mid x_{t}\right) u_{t} \beta_{i}\left(e_{g_{t}}\right) \theta_{i}\left(g_{t+d} \mid x_{t}\right) \\
& =\sum_{j=1}^{M 1} \sum_{i=1}^{M_{2}} \alpha_{j}\left(e_{f_{t}}\right) \beta_{i}\left(e_{g_{t}}\right) \psi_{j i}\left(\mathrm{y}_{t+d} \mid x_{t}, u_{t}\right)
\end{aligned}
$$

where

$$
\psi_{j i}\left(\mathrm{y}_{t+d} \mid x_{t}\right)=\frac{1}{\left(2 \pi \gamma_{j i}\right)^{c / 2}} \exp \left(-\frac{\left\|\mathrm{y}_{t+d}-\overline{\mathrm{y}}_{j i}\left(x_{t}\right)\right\|^{2}}{2 \gamma_{j i}\left(x_{t}\right)}\right),
$$

and where

$$
\begin{aligned}
\gamma_{j i} & =\sigma_{j}^{2}\left(x_{t}\right)+u_{t}^{2} \rho_{i}^{2}\left(x_{t}\right) \\
\bar{y}_{j i} & =\mu_{j}\left(x_{t}\right)+u_{t} \nu_{i}\left(x_{t}\right) .
\end{aligned}
$$


Proof. The proof follows directly from the property that the moment generating function of the sum of independent random variables is the product of the individual moment generating functions, and that the moment generating function of a Gaussian probability distribution function is also of Gaussian form. The details of the proof are provided in Appendix A.

Having obtained the distribution of the system output, the tracking error distribution can be shown to be given by the form specified in the following lemma.

Lemma 3.2. The probability distribution of the tracking error of the system output defined in Equation (4) is given by,

$$
P_{e}\left(e_{t+d} \mid x_{t}, e_{f_{t}}, e_{g_{t}}\right)=\sum_{j=1}^{M 1} \sum_{i=1}^{M_{2}} \alpha_{j}\left(e_{f_{t}}\right) \beta_{i}\left(e_{g_{t}}\right) \Psi_{j i}\left(e_{t+d} \mid x_{t}, u_{t}\right)
$$

where

$$
\Psi_{j i}\left(\mathrm{y}_{t+d} \mid x_{t}\right)=\frac{1}{\left(2 \pi \gamma_{j i}\right)^{c / 2}} \exp \left(-\frac{\left\|\mathrm{y}_{t+d}-\left(\overline{\mathrm{y}}_{j i}\left(x_{t}\right)-\mathrm{y}_{t+d}^{r}\right)\right\|^{2}}{2 \gamma_{j i}\left(x_{t}\right)}\right),
$$

and where $\gamma_{j i}$ and $\overline{\mathrm{y}}_{j i}$ are as defined in Equations (16) and (17) respectively.

Proof. The proof follows directly from Lemma 3.1 and Equation (2).

The above result will naturally lead to the use of stochastic estimation and control algorithms. In the proposed scheme, the derivation of control signals utilizes the probabilistic information from the multiple mode density networks.

\section{Controller Design}

The controller design is concerned with the problem of finding a randomised controller such that the objective functional defined in Equation (3) is minimised. This is a non-linear optimisation problem that can be solved by setting the derivative of the objective functional (3) with respect to the control signal equal to zero,

$$
\frac{\partial J\left(u_{t}\right)}{\partial u_{t}}=0
$$

Before demonstrating the exploitation of Equation (20) for the derivation of the proposed control algorithm, we first discuss various methods proposed in the literature for the selection of the output from an arbitrary distribution of mixture of Gaussians. One method is based on the utilisation of the complete description of the conditional density function of the tracking error as 
stated by equation (18). However, this does not allow for the selection of the individual modes. An alternative as used in this paper is the estimation of the error probability density function using the MMDN where the estimated distribution consists of a number of distinct modes switched by the error dependent priors. This consequently suggests the selection of only one component from the estimated conditional density. One possibility for the selection of the specific component is the selection of the most probable component. The derivation of the optimal control law later, depends on the method used for the selection of the output of the MMDN.

\subsection{Conditional Density Function based PMAC Controller Design}

The evaluation of the objective functional (3) based on the estimated conditional density function of tracking error as stated in Equation (18) yields,

$$
\begin{aligned}
J\left(u_{t}\right) & =R \sum_{j} \sum_{i} \alpha_{j}\left(e_{f_{t}}\right) \beta_{i}\left(e_{g_{t}}\right)\left\{\gamma_{j i}\left(x_{t}\right)+\left[\chi_{j i}\left(x_{t}\right)-\sum_{l_{1}} \sum_{l_{2}} \alpha_{l_{1}}\left(e_{f_{t}}\right) \beta_{l_{2}}\left(e_{g_{t}}\right) \chi_{l_{1} l_{2}}\left(x_{t}\right)\right]^{2}\right\} \\
& +M\left(\sum_{j} \sum_{i} \alpha_{j}\left(e_{f_{t}}\right) \beta_{i}\left(e_{g_{t}}\right) \chi_{j i}\left(x_{t}\right)\right)^{2}+Q u_{t}^{2},
\end{aligned}
$$

where $\chi_{j i}=\mu_{j}\left(x_{t}\right)-\mathrm{y}_{t+d}^{r}+u_{t} \nu_{i}\left(x_{t}\right)$. The first term in this equation is the variance of the tracking error density function. The second term corresponds to the squared mean of the conditional density function of the tracking error. This mean is equivalent to the function computed by a standard network trained using least squares method. The variance however is more general to that computed by least squares method since this variance is constructed to be a general function of the input values, $x_{t}$. Thus, this method in fact reproduces the conventional least squares method as a special case. As such, this method may not be ideal for the derivation of the optimal control law for the switching control problem considered in this paper as will be demonstrated in the simulation example section.

Minimisation of the explicit objective functional (21) leads to the control law specified in the following theorem.

Theorem 4.1. The control law minimising the performance index $J\left(u_{t}\right)$ of Equation (21) subject to the density of the tracking error function of Equation (18) and the density function of the non-linear models of the system dynamics of Equations (9) and (10), is given by

$$
u_{t}^{*}=\frac{M\left(\mathrm{y}_{t+d}^{r}-\hat{f}_{t+d}\left(x_{t}\right)\right) \hat{g}_{t+d}\left(x_{t}\right)}{R s_{g_{t+d}}^{2}\left(x_{t}\right)+Q+M \hat{g}_{t+d}^{2}\left(x_{t}\right)},
$$


where

$$
\begin{aligned}
\hat{f}_{t+d}\left(x_{t}\right) & =\sum_{j} \alpha_{j}\left(e_{f_{t}}\right) \mu_{j}\left(x_{t}\right), \\
\hat{g}_{t+d}\left(x_{t}\right) & =\sum_{i} \beta_{i}\left(e_{g_{t}}\right) \nu_{i}\left(x_{t}\right), \\
s_{g_{t+d}}^{2}\left(x_{t}\right) & =\sum_{i} \beta_{i}\left(e_{g_{t}}\right)\left\{\rho_{i}^{2}\left(x_{t}\right)+\left\|\nu_{i}\left(x_{t}\right)-\sum_{l_{2}} \beta_{l_{2}}\left(e_{g_{t}}\right) \nu_{l_{2}}\left(x_{t}\right)\right\|^{2}\right\} .
\end{aligned}
$$

Proof. The proof follows directly from the substitution of the objective functional of Equation (21) into Equation (20). The proof is given in Appendix B.

\subsection{Most Probable Component based PMAC Controller Design}

For the switching control problem considered in this paper, the most probable component of the tracking error distribution would be more appropriate. Since each component of the mixture of Gaussians in the MMDN is normalised, then under the assumption that the kernels densities are not strongly overlapping, the most probable component can be shown to be given by,

$$
\arg \max _{j, i}\left\{\alpha_{j}\left(e_{f_{t}}\right) \beta_{i}\left(e_{g_{t}}\right)\right\}
$$

This means that one Gaussian model can be available at a specific instant of time,

$$
P_{e}\left(e_{t+d} \mid x_{t}\right)=\frac{1}{\left(2 \pi \gamma_{j i}\right)^{c / 2}} \exp \left(-\frac{\left\|\mathrm{y}_{t+d}-\left(\overline{\mathrm{y}}_{j i}\left(x_{t}\right)-\mathrm{y}_{t+d}^{r}\right)\right\|^{2}}{2 \gamma_{j i}\left(x_{t}\right)}\right),
$$

and where $\gamma_{j i}$ and $\bar{y}_{j i}$ are as defined in Equations (16) and (17) respectively. For the multimodal systems considered in this paper, the most probable component was proven to be more suitable as will be demonstrated in the simulation study. The evaluation of the objective functional (3) based on the estimated most probable component of the tracking error as stated in Equation (24) yields,

$$
J\left(u_{t}\right)=M\left[\mu_{j}\left(x_{t}\right)+\nu_{i}\left(x_{t}\right) u_{t}-\mathrm{y}_{t+d}^{r}\right]^{2}+R\left(\sigma_{j}^{2}\left(x_{t}\right)+\rho_{i}^{2}\left(x_{t}\right) u_{t}^{2}\right)+Q u_{t}^{2},
$$

where the first and second terms respectively represent the square mean and the variance of the most probable Gaussian component. The optimal control law that minimizes the performance function given in Equation (25) is stated in the following theorem. 
Theorem 4.2. The optimal control law that minimizes the performance function $J\left[u_{t}\right]$ of Equation (25) subject to the tracking error conditional density function of Equation (24), is given by

$$
u_{t}^{*}=\frac{M\left[\mathrm{y}_{t+d}^{r}-\mu_{j}\left(x_{t}\right)\right] \nu_{i}\left(x_{t}\right)}{M \nu_{i}^{2}\left(x_{t}\right)+Q+R \rho_{i}^{2}\left(x_{t}\right)} .
$$

Proof. : The proof of Theorem 4.2 can be obtained by evaluating the derivative of the performance measure $J\left[u_{t}\right]$ of Equation (25) with respect to $u_{t}$ and setting the derivative equal to zero.

\section{Simulation example}

In this section a second order affine non-linear system of the form defined in Equation (4) is simulated. The system is described by three modes, where each mode is characterised by a pair of the following equations:

$$
\begin{aligned}
& f_{1}=-\frac{1.5 \mathrm{y}_{t-1} \mathrm{y}_{t-2}}{1+\mathrm{y}_{t-1}^{2}+\mathrm{y}_{t-2}^{2}}+0.35 \sin \left[\mathrm{y}_{t-1}+\mathrm{y}_{t-2}\right], \quad g_{1}=5, \\
& f_{2}=\frac{2.5 \mathrm{y}_{t-1} \mathrm{y}_{t-2}}{1+\mathrm{y}_{t-1}^{2}+\mathrm{y}_{t-2}^{2}}, \quad g_{2}=1, \\
& f_{3}=\frac{1.5 \mathrm{y}_{t-1} \mathrm{y}_{t-2}}{1+\mathrm{y}_{t-1}^{2}+\mathrm{y}_{t-2}^{2}}+0.35 \cos \left[\mathrm{y}_{t-1}+\mathrm{y}_{t-2}\right], \quad g_{3}=3 .
\end{aligned}
$$

This system was used in [Fabri and Kadirkamanathan, 2001] to demonstrate the maximum a posteriori innovations dual control. Here the relative degree of the system, $d=1$, the non-linear functions $\left(f_{i}, g_{i}\right)$ are assumed to be unknown, and the state vector $x_{t}$ as can be deduced from the above equation is given by $\left(\mathrm{y}_{t-1}, \mathrm{y}_{t-2}\right)$. To introduce stochasticity to the system, a noise sampled from a Gaussian distribution with zero mean and 0.001 variance was added to the two non-linear functions $f_{t}$ and $g_{t}$.

The three modes are activated during the time intervals shown in Table 1 . The desired system output is obtained by sampling a unit amplitude, $0.1 \mathrm{~Hz}$ square wave, with a sampling frequency $F_{s}=20 H z$. The square wave is then filtered with a first order low pass filter with a time constant $\tau=0.5 \mathrm{~s}$.

In the first set of experiments, we compare the control results obtained from the most probable system output as evaluated from the multiple mode density network with those obtained from the conditional mean. Two MMDNs were used to estimate the system given by Equation (27). The first MMDN used to estimate the $f_{i}$ 's functions has three Gaassian kernel functions and uses two 
Table 1 . The mode activity of simulated system

\begin{tabular}{|c|c|}
\hline Mode & Intervals of Activity (secs) \\
\hline 1 & {$[0,20),(43,57),(58,100]$} \\
2 & {$[20,29),(71,85]$} \\
3 & {$[29,43],[57,71]$} \\
\hline
\end{tabular}

multi layer perceptron neural networks each with two layers, twenty one tanh sigmoidal units in the hidden layer, and nine outputs. The second MMDN used to estimate the $g_{i}$ 's functions has three Gaussian kernel functions and uses two multi layer perceptron neural networks each with two layers, five tanh sigmoidal units in the hidden layer, and nine outputs. The parameters of the networks were initialised randomly. To ensure fair comparison, both conditional mean and most probable control laws are simulated with the same noise sequence. This experiment was conducted using the design parameters, $M=1, R=0.2$ and $Q=0.0001$. The results of the most probable output based control law are shown in Figure 2. This figure shows that by taking the most probable output of the MMDN components as an estimate for the actual output of the system dynamics, the derived control law which also uses knowledge of the uncertainty in the estimated output are capable of controlling the system output and driving it to accurately track the reference signal. Notice that despite the unknown mode dynamics, the system output is superimposed on the desired output and smoothly tracks the reference signal showing short period transient spikes only following a mode transition. This smooth tracking can be attributed to the fact that the switching probabilities of conditional distribution of the system tracking error as estimated by the MMDN are continuous functions of the error between the kernel components and the currently active mode of the system. Figure 2(b), shows the tracking error $\left(y_{d}-y\right)$ which shows the deviation between the system output and the desired output. Figure 2(c), shows the control input. It can be seen from this figure that despite the mode transition, the control signal changes smoothly with the change in the reference signal. Figure 3 shows the corresponding results obtained from the conditional mean based control law. The figure shows bad tracking performance as can be seen from the large deviations between the system output and the desired output. This confirms that the conditional mean is not suitable for switching control problems where the plant dynamics switch from one mode of operation to another.

The second set of experiments shows the performance of the control system if the exact system functions, $\left(f_{i}, g_{i}\right)$ were used in Equation (26) instead of their estimates $\left(\mu_{j}, \nu_{i}\right)$. In addition, note that as a result of using the exact system functions the term $\rho_{i}^{2}$ in the denominator of Equation (26) will be zero. The results of this experiment are shown in Figure 4. This figure shows very 
similar performance when compared to the performance of the control system, shown in Figure 2(a), if the actual functions were assumed to be unknown and replaced by their estimates $\left(\mu_{j}, \nu_{i}\right)$. The assumption of the availability of the exact system model is unrealistic in practice meaning that a model need to be obtained either from the physical system parameters or from the input and output data of the system.

To show the effect of the design parameters, $M, Q$ and $R$ on the closed loop performance of the control system, we conduct another set of simulation experiments using three different design parameter settings corresponding to heuristic certainty equivalence $R=0$, and dual control with two different design settings: $R=0.5$ and $R=0.2$. The same noise sequence, initial conditions, control penalty $Q=0.0001$, weight of the mean of the tracking error $M=1$, and reference input were used in each case. The result is shown in Figure 5. As expected, the figure shows that the heuristic certainty equivalence controller exhibits large overshoot because it is not taking into consideration the functional uncertainty of the system models. The dual controllers however show similar performance to each other, clearly showing no extra particular overshoot whilst tracking the reference input with a good accuracy. This can be clearly seen from the magnified figures in Figure 5 which show the response of the system in capturing mode 1 .

The last set of experiments compares the performance of the proposed MMDN with the enhanced version of MDN [Herzallah and Lowe, 2003], and the single model standard multi layer perceptron (MLP) neural network that is adapted online to track the variation in the system dynamics. In the enhanced MDN experiment, two MDN's were used to estimate the system functions $\left(f_{i}\right.$ and $\left.g_{i}\right)$. The MDN that estimates the $f_{i}$ functions has three Gaussian Kernel functions and uses a multi layer perceptron neural network with two layers, twenty one tanh sigmoidal units in the hidden layer, and nine outputs. The MDN that estimates the $g_{i}$ functions has three Gaussian kernel functions and uses a multi layer perceptron neural network with two layers, five tanh sigmoidal units in the hidden layer, and nine outputs. For the standard MLP implementation, two MLP networks with eleven and five hidden neurons were used to estimate the $f_{i}$ and $g_{i}$ functions respectively, whose parameters were adapted online to track the variations when the system switches from one mode of operation to another. All model complexities determined by cross validation. The results of both experiments are shown in Figure 6. As expected, both the enhanced version of the MDN and standard MLP show very bad control performance and inability to track the desired system output. Figure 6(a) shows that although a standard MLP can normally track slow varying system parameters, it is inadequate for systems that switch modes abruptly. The results of the enhanced version of MDN as shown in Figure 6(b) also demonstrates bad control performance for the considered jump problem where the currently active mode and its onset do not depend on any of the measurable variables that are 
accessible for estimation.

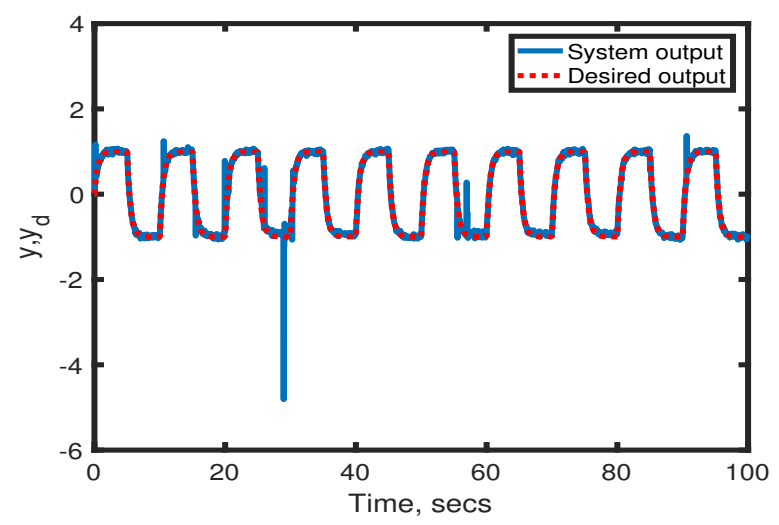

(a)

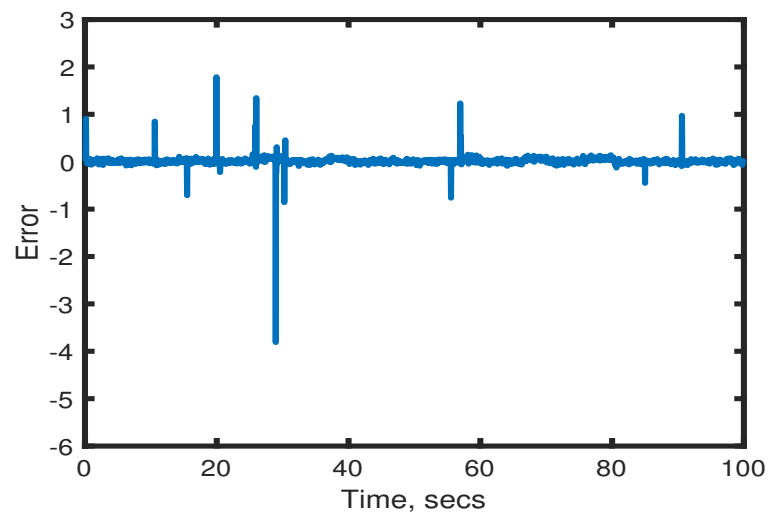

(b)

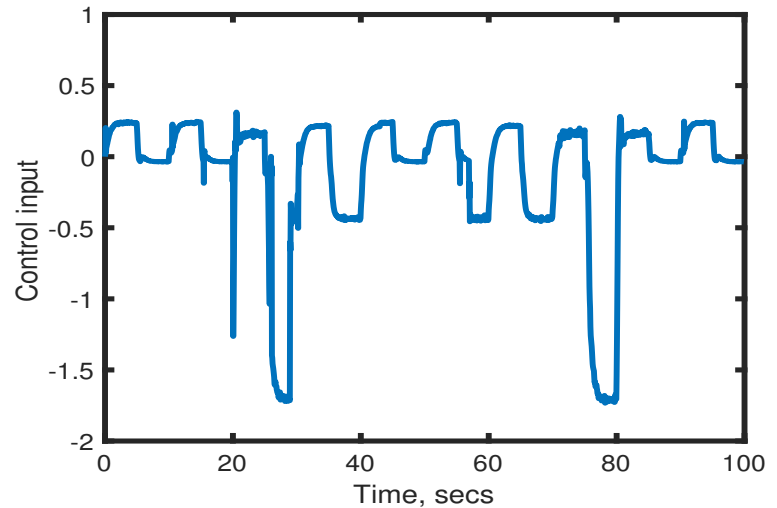

(c)

Figure 2. Most probable control results using the design parameters $M=1, R=0.2$ and $Q=0.0001$ : output, tracking error and control input (a) the actual and reference model outputs of most probable based control. (b) tracking error of most probable based control. (c) control law of most probable based control. 


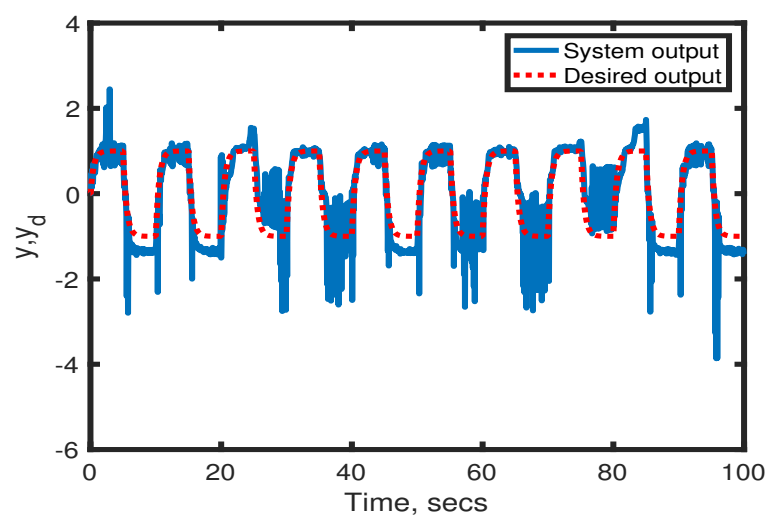

(a)

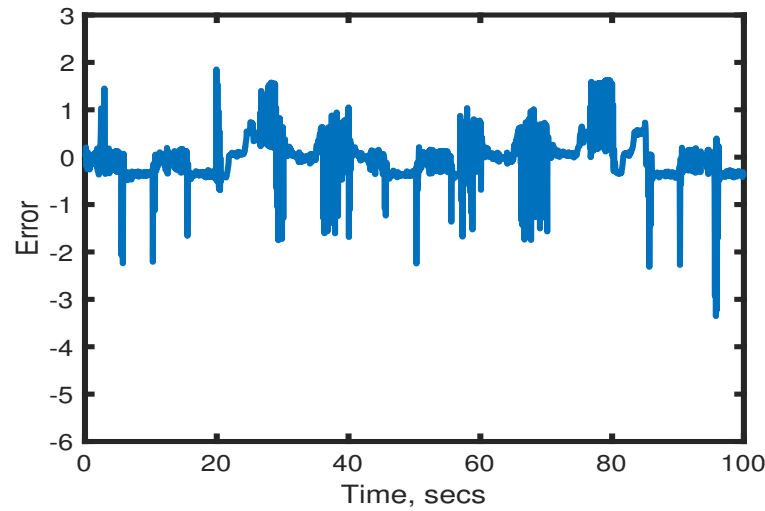

(b)

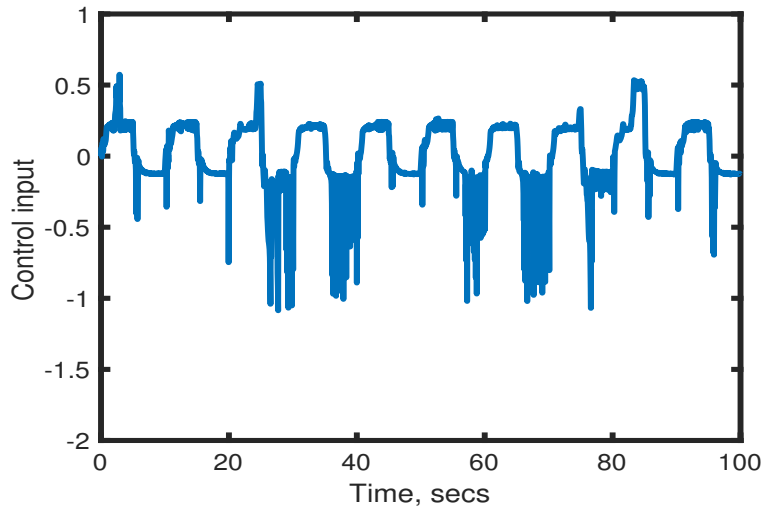

(c)

Figure 3. Conditional mean control results using the design parameters $M=1$, $R=0.2$ and $Q=0.0001$ : output, tracking error and control input(a) the actual and reference model outputs of conditional mean based based control. (b) tracking error of conditional mean based control. (c) control law of conditional mean based control. 


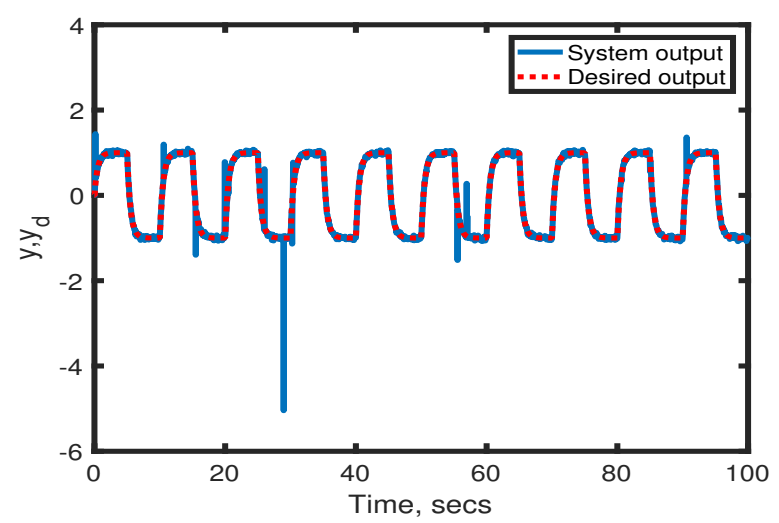

Figure 4. The effect of using the exact system functions, $\left(f_{i}, g_{i}\right)$ in Equation (26) instead of their estimates on the performance of the control system. 


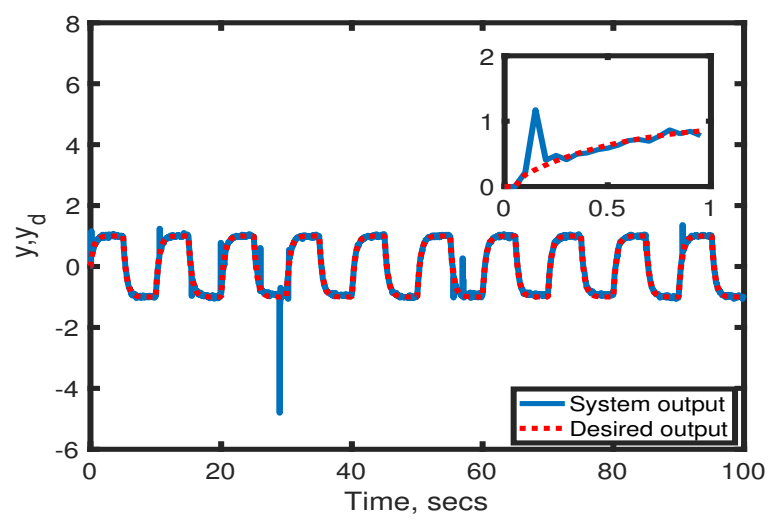

(a)

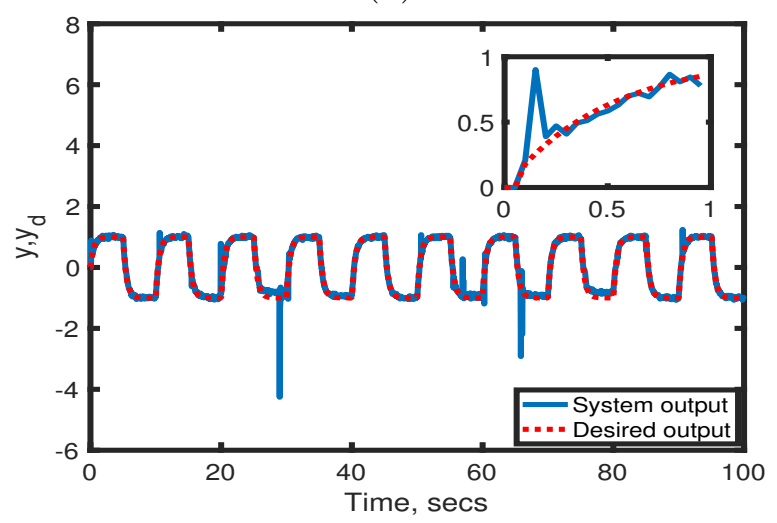

(b)

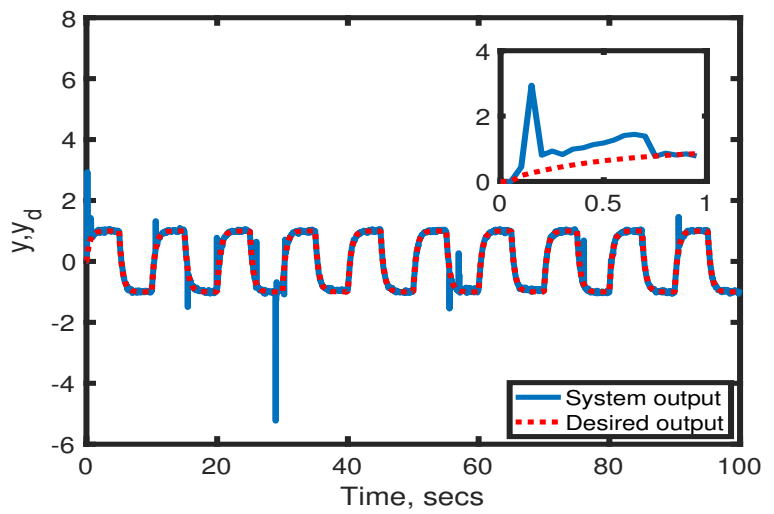

(c)

Figure 5. Most probable control results for three set of design parameters: (a) the actual and reference model outputs of conditional mean based based control using $M=1, R=0.2$ and $Q=0.0001$. (b) the actual and reference model outputs of conditional mean based based control using $M=1, R=0.5$ and $Q=0.0001$. (c) the actual and reference model outputs of conditional mean based based control using $M=1, R=0$ and $Q=0.0001$. 


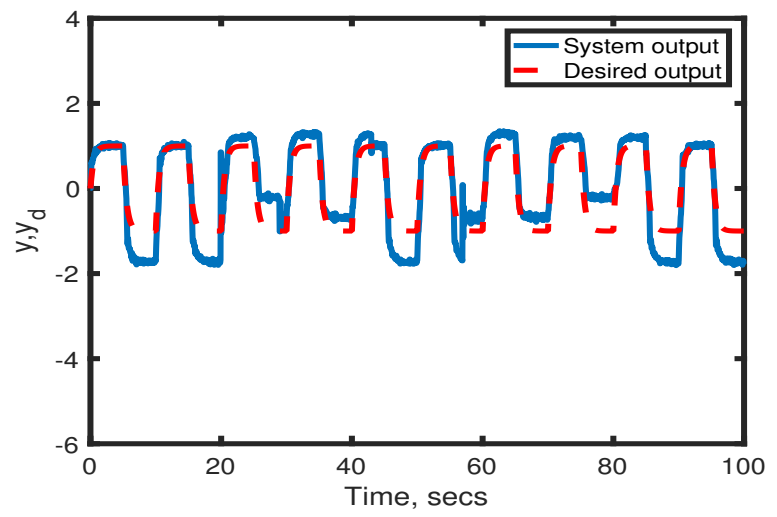

(a)

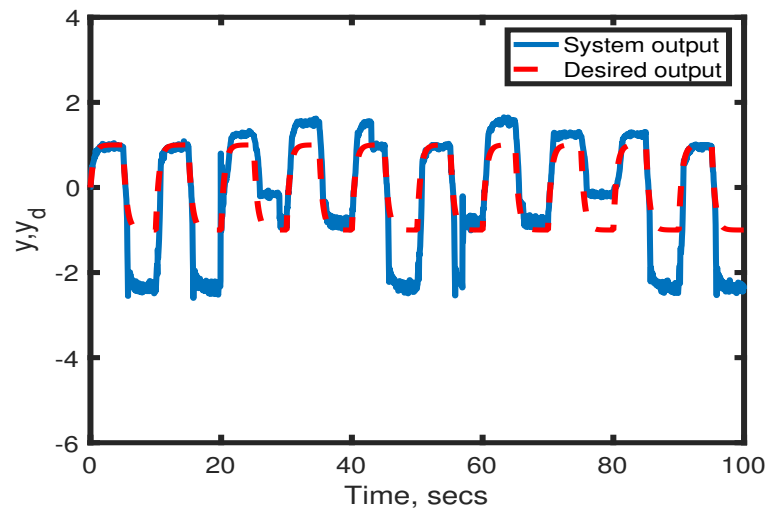

(b)

Figure 6. Standard MLP and enhanced MDN control results: (a) the actual and reference model outputs of the standard MLP based control. (b) the actual and reference model outputs of the enhanced MDN based control. 


\section{Conclusion}

A new probabilistic multimodal adaptive control framework has been proposed. This control framework provides a general solution to the problem of deriving the optimal control law of dynamical systems that switch modes abruptly and arbitrarily in time. Furthermore, to address the multimodality of the system dynamics, we developed a novel architecture called a Multiple Mode Density Network that can estimate an arbitrary distribution of the system dynamics using a mixture of kernel functions. The MMDN model can divide the learning experience among its kernel functions based on the predicted error values between the actual and estimated modes. Following the estimation of the arbitrary distribution of the system dynamics, we then defined the objective function of the control problem such that it utilises the complete information from this distribution. The theoretical findings presented were then validated on a dynamical switching example. The example confirms the effectiveness of the proposed multimodal probabilistic adaptive control framework in achieving good tracking performance for the desired output.

\section{A Convolution of Mixtures of Probability Distributions}

Proposition A.1 The convolution of two independent Gaussian mixture models is also a Gaussian mixture model.

Proof: Specific to the notation of this paper, if $f_{t+d}$ and $g_{t+d}$ are independent random signals, with moment generating functions $M_{f}[s]$ and $M_{g}[s]$, then the moment generating function of a linear combination of the random variable $y_{t+d}=f_{t+d}+u_{t} g_{t+d}$ is simply $M_{y}[s]=M_{f}[s] M_{g}\left[u_{t} s\right]$. The product of two moment generating functions is equivalent to the convolution of the respective probability distributions [Feller, 1971]. Since the distributions of $f_{t+d}$ and $g_{t+d}$ are finite linear combinations of kernel distribution functions, then the moment generating function of a linear combination of distributions is the same linear combination of individual component moment generating functions. ie since

$$
\begin{aligned}
& P\left(f_{t+d} \mid x_{t}, e_{f_{t}}\right)=\sum_{j=1}^{M_{1}} \alpha_{j}\left(e_{f_{t}}\right) \phi_{j}\left(f_{t+d} \mid x_{t}\right), \\
& P\left(g_{t+d} \mid x_{t}, e_{g_{t}}\right)=\sum_{i=1}^{M_{2}} \beta_{i}\left(e_{g_{t}}\right) \theta_{i}\left(g_{t+d} \mid x_{t}\right),
\end{aligned}
$$

then the corresponding moment generating functions are: 


$$
\begin{aligned}
& M_{f}[s]=\sum_{j=1}^{M_{1}} \alpha_{j}\left(e_{f_{t}}\right) M_{\phi_{j}}[s] \\
& M_{g}[s]=\sum_{i=1}^{M_{2}} \beta_{i}\left(e_{g_{t}}\right) M_{\theta_{i}}[s] .
\end{aligned}
$$

Therefore the moment generating function of $y_{t+d}$ is just

$$
M_{y}[s]=\sum_{j=1}^{M_{1}} \sum_{i=1}^{M_{2}} \beta_{i}\left(e_{g_{t}}\right) \alpha_{j}\left(e_{f_{t}}\right) M_{\phi_{j}}[s] M_{\theta_{i}}\left[u_{t} s\right] .
$$

Then it only suffices to show that for Gaussian kernels a product of two Gaussian moment generating functions corresponds to a Gaussian moment generating function, (ie so that $M_{\phi_{j}}[s] M_{\theta_{i}}\left[u_{t} s\right]$ is a Gaussian moment generating function).

This is trivial since the moment generating function of a normal random variable $X$ is:

$$
M_{X}[s]=\int_{-\infty}^{\infty} \exp (s x) \frac{1}{\sqrt{2 \pi} \sigma} \exp \left(-\frac{x^{2}}{2 \sigma^{2}}\right) d x=\exp \left(\frac{s^{2} \sigma^{2}}{2}+\mu_{x} s\right) .
$$

Therefore for two independent normal random variables $X_{1}$ and $X_{2}$ with parameters $\left(\mu_{1}, \sigma_{1}^{2}\right)$ and $\left(\mu_{2}, \sigma_{2}^{2}\right)$, then

$$
M_{X_{1}+X_{2}}=\exp \left(\frac{\left(\sigma_{1}^{2}+\sigma_{2}^{2}\right) s^{2}}{2}+\left(\mu_{1}+\mu_{2}\right) s\right)
$$

which is also the moment generating function of the normal distribution with parameters $\left(\mu_{1}+\mu_{2}, \sigma_{1}^{2}+\sigma_{2}^{2}\right)$.

Converting back to the original variables

$$
M_{\phi_{j}}[s] M_{\theta_{i}}\left[u_{t} s\right] \equiv M_{\psi_{i j}}[s]
$$

where

$$
M_{\psi_{i j}}[s]=\exp \left(\frac{\left(\sigma_{j}^{2}+u_{t}^{2} \rho_{i}^{2}\right) s^{2}}{2}+\left(\mu_{j}+u_{t} \nu_{i}\right) s\right)
$$

Combining these observations it is a trivial generalisation to map over to the kernel mixture models of Equations (9) and (10) in which the individual convolutions between the Gaussian mixture components are weighted by the coefficients $\alpha_{j}\left(e_{f_{t}}\right), \beta_{i}\left(e_{g_{t}}\right)$. The resultant distribution is therefore a mixture combination of Gaussian distributions, ie. the distribution of the random variable $y_{t+d} \equiv f_{t+d}+u_{t} g_{t+d}$ can be expressed as a recombined Gaussian mixture 
model:

$$
p\left(\mathrm{y}_{t+d} \mid x_{t}, e_{f_{t}}, e_{g_{t}}\right)=\sum_{j=1}^{M 1} \sum_{i=1}^{M_{2}} \alpha_{j}\left(e_{f_{t}}\right) \beta_{i}\left(e_{g_{t}}\right) \psi_{j i}\left(\mathrm{y}_{t+d} \mid x_{t}\right)
$$

where

$$
\psi_{j i}\left(\mathrm{y}_{t+d} \mid x_{t}\right)=\frac{1}{\left(2 \pi \gamma_{j i}\right)^{c / 2}} \exp \left(-\frac{\left\|\mathrm{y}_{t+d}-\overline{\mathrm{y}}_{j i}\left(x_{t}\right)\right\|^{2}}{2 \gamma_{j i}\left(x_{t}\right)}\right),
$$

and where

$$
\begin{aligned}
\gamma_{j i} & =\sigma_{j}^{2}\left(x_{t}\right)+u^{2}(t) \rho_{i}^{2}\left(x_{t}\right) \\
\overline{\mathrm{y}}_{j i} & =\mu_{j}+u(t) \nu_{i} .
\end{aligned}
$$

\section{B Optimal Control Law}

The objective functional of the optimal control problem is defined in (21), repeated here,

$$
\begin{aligned}
J\left(u_{t}\right) & =R \sum_{j} \sum_{i} \alpha_{j}\left(e_{f_{t}}\right) \beta_{i}\left(e_{g_{t}}\right)\left\{\gamma_{j i}\left(x_{t}\right)+\left[\chi_{j i}\left(x_{t}\right)-\sum_{l_{1}} \sum_{l_{2}} \alpha_{l_{1}}\left(e_{f_{t}}\right) \beta_{l_{2}}\left(e_{g_{t}}\right) \chi_{l_{1} l_{2}}\left(x_{t}\right)\right]^{2}\right\} \\
& +M\left(\sum_{j} \sum_{i} \alpha_{j}\left(e_{f_{t}}\right) \beta_{i}\left(e_{g_{t}}\right) \chi_{j i}\left(x_{t}\right)\right)^{2}+Q u_{t}^{2}
\end{aligned}
$$

where,

$$
\begin{aligned}
\gamma_{j i} & =\sigma_{j}^{2}\left(x_{t}\right)+u_{t}^{2} \rho_{i}^{2}\left(x_{t}\right), \\
\chi_{j i} & =\bar{\mu}_{j}+u_{t} \nu_{i},
\end{aligned}
$$

and where

$$
\bar{\mu}_{j}=\mu_{j}-\mathrm{y}_{t+d}^{r} .
$$

Taking the derivative of the objective functional (B.1) with respect to the control signal and setting it to zero yields, 


$$
\begin{aligned}
0 & =R \sum_{j} \sum_{i} \alpha_{j}\left(e_{f_{t}}\right) \beta_{i}\left(e_{g_{t}}\right) u_{t} \rho_{i}^{2} \\
& +R \sum_{j} \sum_{i} \alpha_{j}\left(e_{f_{t}}\right) \beta_{i}\left(e_{g_{t}}\right)\left[\chi_{j i}\left(x_{t}\right)-\sum_{l_{1}} \sum_{l_{2}} \alpha_{l_{1}}\left(e_{f_{t}}\right) \beta_{l_{2}}\left(e_{g_{t}}\right) \chi_{l_{1} l_{2}}\left(x_{t}\right)\right] \\
& {\left[\nu_{i}-\sum_{l_{1}} \sum_{l_{2}} \alpha_{l_{1}}\left(e_{f_{t}}\right) \beta_{l_{2}}\left(e_{g_{t}}\right) \nu_{l_{2}}\right]+M\left(\sum_{j} \sum_{i} \alpha_{j}\left(e_{f_{t}}\right) \beta_{i}\left(e_{g_{t}}\right)\right)^{2} \nu_{i}\left(\bar{\mu}_{j}+u_{t} \nu_{i}\right) } \\
& +Q u_{t}, \\
& =R u_{t} \sum_{j} \sum_{i} \alpha_{j}\left(e_{f_{t}}\right) \beta_{i}\left(e_{g_{t}}\right) \rho_{i}^{2} \\
& +R \sum_{j} \sum_{i} \alpha_{j}\left(e_{f_{t}}\right) \beta_{i}\left(e_{g_{t}}\right)\left[\bar{\mu}_{j}+u_{t} \nu_{i}\right]\left[\nu_{i}-\sum_{l_{1}} \sum_{l_{2}} \alpha_{l_{1}}\left(e_{f_{t}}\right) \beta_{l_{2}}\left(e_{g_{t}}\right) \nu_{l_{2}}\right] \\
& -R \sum_{j} \sum_{i} \alpha_{j}\left(e_{f_{t}}\right) \beta_{i}\left(e_{g_{t}}\right) \sum_{l_{1}} \sum_{l_{2}} \alpha_{l_{1}}\left(e_{f_{t}}\right) \beta_{l_{2}}\left(e_{g_{t}}\right)\left[\bar{\mu}_{l_{1}}+u_{t} \nu_{l_{2}}\right]\left[\nu_{i}-\sum_{l_{1}} \sum_{l_{2}} \alpha_{l_{1}}\left(e_{f_{t}}\right) \beta_{l_{2}}\left(e_{g_{t}}\right) \nu_{l_{2}}\right] \\
& +M\left(\sum_{j} \sum_{i} \alpha_{j}\left(e_{f_{t}}\right) \beta_{i}\left(e_{g_{t}}\right)\right)^{2} \nu_{i}\left(\bar{\mu}_{j}+u_{t} \nu_{i}\right)+Q u_{t} .
\end{aligned}
$$

Rearranging this equation such that all control signal dependent terms are kept on one side of the equation and all other terms are taken to the other side yields,

$$
\begin{aligned}
& u_{t}\left\{R \sum_{j} \sum_{i} \alpha_{j}\left(e_{f_{t}}\right) \beta_{i}\left(e_{g_{t}}\right) \rho_{i}^{2}+R \sum_{j} \sum_{i} \alpha_{j}\left(e_{f_{t}}\right) \beta_{i}\left(e_{g_{t}}\right) \nu_{i}\left[\nu_{i}-\sum_{l_{1}} \sum_{l_{2}} \alpha_{l_{1}}\left(e_{f_{t}}\right) \beta_{l_{2}}\left(e_{g_{t}}\right) \nu_{l_{2}}\right]\right. \\
- & R \sum_{j} \sum_{i} \alpha_{j}\left(e_{f_{t}}\right) \beta_{i}\left(e_{g_{t}}\right) \sum_{l_{1}} \sum_{l_{2}} \alpha_{l_{1}}\left(e_{f_{t}}\right) \beta_{l_{2}}\left(e_{g_{t}}\right) \nu_{l_{2}}\left[\nu_{i}-\sum_{l_{1}} \sum_{l_{2}} \alpha_{l_{1}}\left(e_{f_{t}}\right) \beta_{l_{2}}\left(e_{g_{t}}\right) \nu_{l_{2}}\right] \\
+ & \left.M\left(\sum_{j} \sum_{i} \alpha_{j}\left(e_{f_{t}}\right) \beta_{i}\left(e_{g_{t}}\right)\right)^{2} \nu_{i}^{2}+Q\right\} \\
= & R \sum_{j} \sum_{i} \alpha_{j}\left(e_{f_{t}}\right) \beta_{i}\left(e_{g_{t}}\right) \sum_{l_{1}} \sum_{l_{2}} \alpha_{l_{1}}\left(e_{f_{t}}\right) \beta_{l_{2}}\left(e_{g_{t}}\right) \bar{\mu}_{l_{1}}\left[\nu_{i}-\sum_{l_{1}} \sum_{l_{2}} \alpha_{l_{1}}\left(e_{f_{t}}\right) \beta_{l_{2}}\left(e_{g_{t}}\right) \nu_{l_{2}}\right] \\
- & R \sum_{j} \sum_{i} \alpha_{j}\left(e_{f_{t}}\right) \beta_{i}\left(e_{g_{t}}\right) \bar{\mu}_{j}\left[\nu_{i}-\sum_{l_{1}} \sum_{l_{2}} \alpha_{l_{1}}\left(e_{f_{t}}\right) \beta_{l_{2}}\left(e_{g_{t}}\right) \nu_{l_{2}}\right] \\
- & M\left(\sum_{j} \sum_{i} \alpha_{j}\left(e_{f_{t}}\right) \beta_{i}\left(e_{g_{t}}\right)\right)^{2} \nu_{i} \bar{\mu}_{j} .
\end{aligned}
$$

Since $\alpha_{j}\left(e_{f_{t}}\right)$ and $\beta_{i}\left(e_{g_{t}}\right)$ are prior probabilities, this means that

$$
\sum_{j} \alpha_{j}\left(e_{f_{t}}\right)=1, \quad \sum_{i} \beta_{i}\left(e_{g_{t}}\right)=1 .
$$

Using this equation in Equation (B.6) and simplifying yields, 


$$
\begin{aligned}
& u_{t}\left\{R \sum_{i} \beta_{i}\left(e_{g_{t}}\right) \rho_{i}^{2}+R \sum_{i} \beta_{i}\left(e_{g_{t}}\right) \nu_{i}\left[\nu_{i}-\sum_{l_{2}} \beta_{l_{2}}\left(e_{g_{t}}\right) \nu_{l_{2}}\right]\right. \\
- & \left.R \sum_{i} \beta_{i}\left(e_{g_{t}}\right) \sum_{l_{2}} \beta_{l_{2}}\left(e_{g_{t}}\right) \nu_{l_{2}}\left[\nu_{i}-\sum_{l_{2}} \beta_{l_{2}}\left(e_{g_{t}}\right) \nu_{l_{2}}\right]+M\left(\sum_{i} \beta_{i}\left(e_{g_{t}}\right) \nu_{i}\right)^{2}+Q\right\} \\
= & R \sum_{i} \beta_{i}\left(e_{g_{t}}\right) \sum_{l_{1}} \alpha_{l_{1}}\left(e_{f_{t}}\right) \bar{\mu}_{l}\left[\nu_{i}-\sum_{l_{2}} \beta_{l_{2}}\left(e_{g_{t}}\right) \nu_{l_{2}}\right] \\
- & R \sum_{j} \alpha_{j}\left(e_{f_{t}}\right) \bar{\mu}_{j} \sum_{i} \beta_{i}\left(e_{g_{t}}\right)\left[\nu_{i}-\sum_{l_{2}} \beta_{l_{2}}\left(e_{g_{t}}\right) \nu_{l_{2}}\right] \\
- & M \sum_{i} \beta_{i}\left(e_{g_{t}}\right) \nu_{i} \sum_{j} \alpha_{j}\left(e_{f_{t}}\right) \bar{\mu}_{j} .
\end{aligned}
$$

The first two terms on the right hand side of Equation (B.8) then vanish as they are equal but have opposite signs. Hence Equation (B.8) simplifies to the form,

$$
\begin{aligned}
& u_{t} R \sum_{i} \beta_{i}\left(e_{g_{t}}\right)\left\{\rho_{i}^{2}+\nu_{i}^{2}-2 \nu_{i} \sum_{l_{2}} \beta_{l_{2}}\left(e_{g_{t}}\right) \nu_{l_{2}}+\left(\sum_{l_{2}} \beta_{l_{2}}\left(e_{g_{t}}\right) \nu_{l_{2}}\right)^{2}\right\} \\
+ & M\left(\sum_{i} \beta_{i}\left(e_{g_{t}}\right) \nu_{i}\right)^{2} u_{t}+Q u_{t} \\
= & -M \sum_{i} \beta_{i}\left(e_{g_{t}}\right) \nu_{i} \sum_{j} \alpha_{j}\left(e_{f_{t}}\right) \bar{\mu}_{j} .
\end{aligned}
$$

If we define,

$$
\begin{aligned}
\hat{f}_{t+d} & =\sum_{j} \alpha_{j}\left(e_{f_{t}}\right) \mu_{j}, \\
\hat{g}_{t+d} & =\sum_{i} \beta_{i}\left(e_{g_{t}}\right) \nu_{i} \\
s_{g_{t+d}}^{2}\left(x_{t}\right) & =\sum_{i} \beta_{i}\left(e_{g_{t}}\right)\left\{\rho_{i}^{2}\left(x_{t}\right)+\left\|\nu_{i}\left(x_{t}\right)-\sum_{l_{2}} \beta_{l_{2}}\left(e_{g_{t}}\right) \nu_{l_{2}}\left(x_{t}\right)\right\|^{2}\right\},
\end{aligned}
$$

then Equation (B.9) can be rewritten as,

$$
\begin{aligned}
& u_{t} R s_{g_{t}}^{2}\left(x_{t}\right)+M\left(\sum_{i} \beta_{i}\left(e_{g_{t}}\right) \nu_{i}\right)^{2} u_{t}+Q u_{t} \\
= & M \sum_{i} \beta_{i}\left(e_{f_{t}}\right) \nu_{i} \sum_{j} \alpha_{j}\left(e_{f_{t}}\right)\left(y_{t+d}^{r}-\mu_{j}\right) .
\end{aligned}
$$




\section{References}

[Apkarian et al. 2014] Apkarian, P., Gahinet, P., \& Buhr, C., (2014), Multi-model, multi-objective tuning of fixed-structure controllers. 2014 European Control Conference (ECC), Jun 24-27. Strasbourg, France. 856-861.

[Bishop, 1995] Bishop, C. M., (1995), Neural Networks for Pattern Recognition. Oxford University Press, New York, N.Y.

[Boskovic and Mehra, 2018] Boškovic, J.D., \& Mehra, R.K., (2002), Multiple-Model Adaptive Flight Control Scheme for Accommodation of Actuator Failures. Journal of Guidance, Control, and Dynamics, 25(4): 712-724.

[Duarte, 1996] Duarte, M., \& Narendra, K., (1996), Indirect Model Reference Adaptive Control with Dynamic Adjustment of Parameters. Int. J. Adapt. Control Signal Process, 10: 603-621.

[Fabri and Kadirkamanathan, 2001] Fabri, S. G, \& Kadirkamanathan, V., (2001), Functional Adaptive Control: An Intelligent Systems Approach. Springer-Verlag.

[Fekri et al., 2006] Fekri, S., Athans, M., \& Pascoal, A., (2006), Issues, progress and new results in robust adaptive control. International Journal of Adaptive Control and Signal Processing, 20: 519-579.

[Feller, 1971] Feller,W., (1971), An Introduction to Probability Theory and Its Applications, John Wiley Sons, New York, Vol. II.

[Fiacchini et al., 2016] iacchini, M., Girard, A., \& Jungers, M., (2016), On the stabilizability of discrete-time switched linear systems: Novel conditions and comparisons. IEEE Transactions on Automatic Control, 61(5): 1181-1193.

[Fiacchini and Tarbouriech, 2017] Fiacchini, M., \& Tarbouriech, S., (2017), Control co-design for discrete-time switched linear systems. Automatica, 82: 181-186.

[Forbes et al., 2004] Forbes, S. G., Forbes,J. F., \& Guay, M., (2004), Control design for first order processes: Shaping the probability density of the process state. Journal of Process Control, 14(4):399-410.

[Fu and Barmish, 1986] Fu, M., \& Barmish, B. R., (1986), Adaptive stabilisation of linear systems via switching control. IEEE Transactions on Automatic Control, 31: 1097-1103.

[Han and Narendra, 2012] Han, Z., \& Narendra, K. S., (2012), New concepts in adaptive control using multiple models. IEEE Transactions on Automatic Control, 57(1): 78-89.

[Herzallah, 2011] Herzallah, R., (2011), Enhancing the performance of intelligent control systems in the face of higher levels of complexity and uncertainty. International Journal of Modelling Identification and Control, 12(4):311-327.

[Herzallah, 2012] Herzallah, R., (2012), Probabilistic control for uncertain systems. Journal of Dynamic Systems, Measurement and Control, 134(2). 
[Herzallah and Lowe, 2003] Herzallah, R., \& Lowe, D., (2003), Robust control of stochastic systems by modelling conditional distributions of control signals. Neural Computing and Applications, 12(2):98-108.

[Hespanha et al., 2003] Hespanha, J. P., Liberzon, D., \& Morse, A. S., (2003), Hysteresis-based switching algorithms for supervisory control of uncertain systems. Automatica, 39: 263-272.

[Kersting, 2017] Kersting, S., \& Buss, M., (2017), Direct and Indirect Model Reference Adaptive Control for Multivariable Piecewise Affine Systems. IEEE Transactions on Automatic Control, 62: 5634-5649.

[Leith et al., 2003] Leith, D. J., Shorten, R. N., Leithead, W. E., Mason, O., \& Curran, P., (2003), Issues in the design of switched linear control systems: A benchmark study. International Journal of Adaptive Control and Signal Processing, 17(2): 103-118.

[Liu et al., 2018] Liu, C., Jiang, B., \& Zhang, K., (2018), Integrated multiplemodel adaptive fault identification and reconfigurable fault-tolerant control for Lead-Wing close formation systems. International Journal of Systems Science, 49(4):701-717.

[Middleton et al., 1988] Middleton, R. H., Goodwin,G. C., Hill,D. J., \& Mayne, D. Q., (1988), Design issues in adaptive control. IEEE Transactions on Automatic Control, 33: 50-58.

[Morse, 1996] Morse, A. S., (1996), Supervisory control of families of linear set-point controllers, part 1: exact matching. IEEE Transactions on Automatic Control, 41: $1413-1431$.

[Morse, 1997] Morse, A. S., (1997), Supervisory control of families of linear setpoint controllers, part 2: robustness. IEEE Transactions on Automatic Control, 42: $1500-1515$.

[Narendra et al., 1995] Narendra, K. S., Balakrishnan, J., \& Ciliz, M. K., (1995), Adaptation and learning using multiple models, switching, and tuning. IEEE Control Systems, 15(3): 37-51.

[Narendra and Chen, 2000] Narendra, K. S. \& Cheng Xiang, (2000), Adaptive control of discrete-time systems using multiple models. IEEE Transactions on Automatic Control, 45(9): 1669-1686.

[Peitz and Dellnitz, 2018] Peitz, S., \& Dellnitz, M., (2018), A survey of recent trends in multiobjective optimal control-surrogate models, feedback control and objective reduction. Mathematical and Computational Applications, 23(30): 133 .

[Piguet et al., 1997] Piguet, Y., Holmberg, U., \& R. Longchamp, (1997), Multiobjective controller design using multiple models. IFAC Robust Control Design, Hungary. 225-230.

[Safonov and Tsao, 1997] Safonov, M. G., \& Tsao, T., (1997), The unfalsified control concept and learning. IEEE Transactions on Automatic Control, 42(6): 843-847. 
[Watanabe and Tzafestas, 1989] Watanabe, K., \& TzafestasS. G., (1989), Multiplemodel adaptive control for jump-linear stochastic systems. International Journal of Control, 50: 1603-1617.

[Yao et al., 2008] Yao,L., Wang, A., \& Wang, H., (2008), Fault detection, diagnosis and tolerant control for non-Gaussian stochastic distribution systems using a rational square root approximation model, International Journal of Modelling Identification and Control, 3(2):162-172.

[Zhang, 2012] Zhang, W., (2012), Stable weighted multiple model adaptive control with improved convergence rate. Proceedings of the 7th IFAC Symposium on Robust Control Design, Aalborg, Denmark. June 20-22.

[Zhang et al., 2012] Zhang, W., Hu, J., \& Abate, A., (2012), Infinite-horizon switched LQR problems in discrete time: a suboptimal algorithm with performance analysis. IEEE Transactions on Automatic Control, 57(7): 1815$-1821$.

[Zhivoglyadov et al., 2000] Zhivoglyadov, P., Middleton, R. H., \& Ful, M.. Localisation based switching adaptive control for time-varying discrete-time systems. IEEE Transactions on Automatic Control, 45(4): 52-75. 